Original Paper

\title{
One-Dimensional Analysis of Full Load Draft Tube Surge Considering the Finite Sound Velocity in the Penstock
}

\author{
Changkun Chen ${ }^{1}$, Christophe Nicolet ${ }^{2}$, Koichi Yonezawa ${ }^{1}$, Mohamed Farhat ${ }^{2}$, Francois Avellan ${ }^{2}$ \\ and Yoshinobu Tsujimoto ${ }^{1}$
}

${ }^{1}$ Graduate School of Engineering Science, Osaka University

Machikaneyama 1-3, Toyonaka, 560-8531, Japan

${ }^{2}$ Laboratory for Hydraulic Machines, EPFL - Swiss Federal Institute of Technology

33 bis av. de Cour, Lausanne, $\mathrm{CH}-1007$, Switerland

\begin{abstract}
The effects of acoustic modes in the penstock on the self-excited oscillation in hydraulic power system were studied by assuming a finite sound velocity in the penstock. The flow in the draft tube is considered to be incompressible assuming that the length of the draft tube is smaller than the wavelength of the oscillation. It was found that various acoustic modes in the penstock can become unstable (amplified) by the diffuser effect of the draft tube or the effect of swirl flow from the runner. Their effects on each mode are discussed.
\end{abstract}

Keywords: Draft tube surge, finite sound velocity, higher order frequencies

\section{Introduction}

Draft tube surge at off-design conditions is one of the most important phenomena in Francis turbines. At part load operation, it has been clarified that it is caused by the resonance of hydraulic system with the precession motion of helical vortex rope in the draft tube ${ }^{[2]-[5]}$. At full load operation, the shape of the vortex changes to an elongated, torch-like structure. And the dynamics of the vortex rope can act as an energy source, leading to the development of self-exited oscillations and dynamic instability within the hydraulic system ${ }^{[6]}[7]$. The cause of the self-excited surge was not clear. References [6] and [7] show that full load surge can be successfully simulated by using appropriate values of mass flow gain factor and cavitation compliance to represent the cavitation effect in the draft tube. However, the flow mechanism determining the value of mass flow gain factor was not clear. In the previous study [1], a one dimensional analytical model was built to study the mechanism of full load draft tube surge, assuming an incompressible flow in the penstock. It was found that the diffuser effect of the draft tube has a destabilizing effect over all flow rates, while the swirl effects stabilize/destabilize the system at larger/smaller flow rates than the swirl free flow rate.

Transient phenomena have been computed for years on hydraulic circuits with turbomachines. Pipeline resonances with the acoustic modes of the inlet or discharge piping can occur when one of the excitation frequencies produced by the turbine happens to coincide with one of the acoustic modes of the system. The pressure oscillation magnitudes associated with these resonances can often cause substantial damage ${ }^{[8]}$. The present study was carried out by considering the effect of finite sound velocity in the penstock based on the original analytical model. The acoustic modes of the penstock were clarified and the influence from diffuser and swirl effect were discussed.

\section{Analytical model and equations}

Figure 1 shows the hydraulic system considered in the present study. The model is composed of an inlet pipe of length $L_{i}$ and area $A_{i}$, a turbine runner, and a draft tube with the inlet and exit areas $A_{c}$ and $A_{e}$, respectively. In real plants, the length of the inlet pipe or penstock is generally larger than the wavelength of the disturbance and the case with finite sound velocity in the penstock is considered. On the other hand, it is expected that the length of the draft tube is smaller than the wavelength. For simplicity, an incompressible flow is assumed in the draft tube.

A cavity of volume $V_{c}$ is assumed downstream of the turbine and upstream of the draft tube. Then, the continuity equation between upstream and downstream flow rates $Q_{1}$ and $Q_{2}$ is: 
By applying Bernoulli's equation to the draft tube, it is obtained,

$p_{a}=p_{\text {exit }}+\rho \frac{L_{e}}{A_{e}} \frac{d Q_{2}}{d t}+\rho \frac{\zeta_{2}-D}{2 A_{e}^{2}} Q_{2}^{2}$

where $L_{e}=\int\left(A_{e} / A(s)\right) d s$ is the effective length of the draft tube, $D=\left(A_{e} / A_{c}\right)^{2}-1$ the diffuser factor, $\zeta_{2}$ the loss coefficient of the draft tube.For simplicity, $\zeta_{2}$ is assumed to be constant although it may depend on the swirl of the discharge flow ${ }^{[9]}$.

At off-design operating point, the discharge flow from the runner swirls and a vortex is formed. If the pressure $p_{c}$ at the vortex center is lower than the vapor pressure, a cavity will appear. The cavity volume is considered to be a function of the core pressure $p_{c}$,

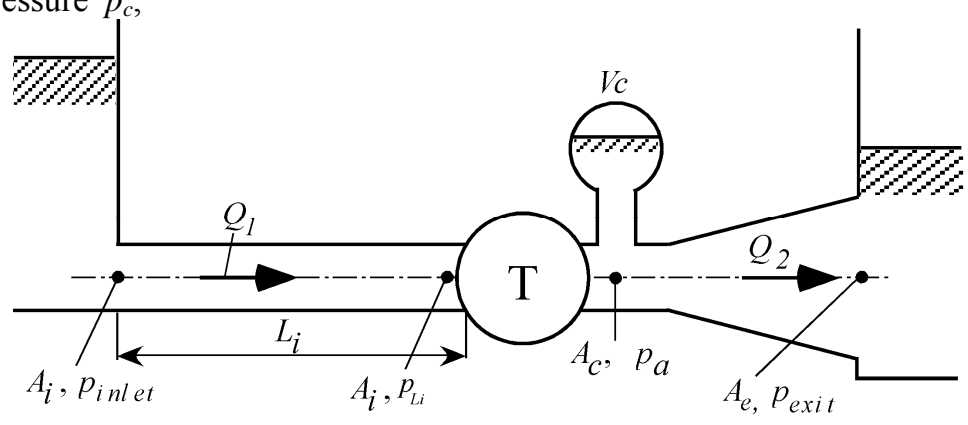

Fig. 1 Hydraulic system for the analysis

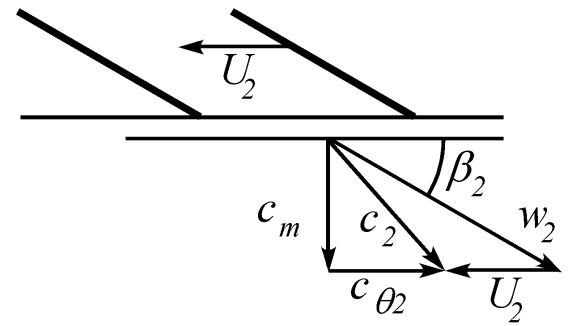

Fig. 2 Velocity triangle at the runner exit

$V_{C}=V_{c}\left(p_{c}\right)$

Due to the centrifugal force on the swirling flow, the core pressure $p_{c}$ is lower than the ambient pressure $p_{a}$

$$
p_{c}=p_{a}-\rho \alpha c_{\theta 2}^{2}
$$

Here, $c_{\theta 2}$ is a representative swirl velocity and $\alpha$ is a pressure coefficient for the swirl effects. If assuming a Rankine's combined vortex with the core radius $r$ and the outer radius $R, \alpha$ is determined to be $\alpha=(R / r)^{2}-1 / 2$, with $c_{\theta 2}$ evaluated at the outer radius $R$, although the real flow from the runner is much more complicated ${ }^{[9]}$.

From the velocity triangle at the runner discharge as shown in Fig. 2, the swirl velocity $c_{\theta 2}$ is:

$$
c_{\theta 2}=c_{m 2} \cot \beta_{2}-U_{2}=\frac{Q_{1}}{S} \cot \beta_{2}-U_{2}
$$

where $\beta_{2}, S$ and $U_{2}$ are vane angle, area and peripheral speed at the runner exit.

By putting Eqs.(2) and (5) in Eq.(4), the cavity core pressure $p_{c}$ can be expressed as:

$$
p_{c}=p_{\text {exit }}+\rho \frac{L_{e}}{A_{e}} \frac{d Q_{2}}{d t}+\rho \frac{\zeta_{2}-D}{2 A_{e}^{2}} Q_{2}^{2}-\rho \alpha\left(\frac{\cot \beta_{2}}{S} Q_{1}-U_{2}\right)^{2}
$$

The cavitation compliance $C$ is defined by

$$
C=-d V_{c} / d p_{C}
$$

Then, the continuity equation (1) can be expressed as

$$
\begin{aligned}
& Q_{2}-Q_{1}=d V_{c} / d t=\left(d V_{c} / d p_{C}\right)\left(d p_{c} / d t\right)=-C\left(d p_{c} / d t\right) \\
& =-\rho C \frac{L_{e}}{A_{e}} \frac{d^{2} Q_{2}}{d t^{2}}+\rho C \frac{D-\zeta_{2}}{A_{e}^{2}} Q_{2} \frac{d Q_{2}}{d t}+2 \rho C \alpha \frac{\cot \beta_{2}}{S}\left(\frac{\cot \beta_{2}}{S} Q_{1}-U_{2}\right) \frac{d Q_{1}}{d t}
\end{aligned}
$$

The second term with $d Q_{2} / d t$ represents the diffuser effect corresponding to the mass flow gain factor. When the discharge flow $Q_{2}$ is increased, the ambient pressure $p_{a}$ is decreased if the diffuser effect $D$ is larger than the loss $\zeta_{2}$, resulting in an increase in cavity volume, which would promote further increase of $Q_{2}$. The third term with $d Q_{1} / d t$ represents the swirl effect. This term may also be called "mass flow gain factor" but is associated with the upstream flow $Q_{1}$. At flow rates lower than design $\left(Q_{1}<Q_{s f}\right.$ $=U_{2} S \tan \beta_{2}$ ), the tangential velocity $c_{\theta 2}$ and the cavity volume decrease as the upstream flow rates $Q_{1}$ is increased, which would promote further increase in $Q_{1}$. Equations (1) to (8) have be derived in Ref.[1] but reproduced here for completeness.

Next, the flow in penstock is considered. Considering the compressibility of the fluid and the elasticity of pipe wall, the momentum and continuity equations are represented by

$$
\begin{aligned}
& \frac{\partial u}{\partial t}+\frac{1}{\rho} \frac{\partial p}{\partial x}=0 \\
& \frac{\partial p}{\partial t}+\rho a^{2} \frac{\partial u}{\partial x}=0
\end{aligned}
$$

where $a$ is the wave speed and can be evaluated from the speed of sound and the geometry of penstock section. The convective term has been ignored as being small with respect to other terms.

By taking the partial derivative of Eq. (10) with respect to $t$ and Eq. (9) with respect to $x$, one may eliminate $u$, which yields

$$
\frac{\partial^{2} p}{\partial t^{2}}-a^{2} \frac{\partial^{2} p}{\partial x^{2}}=0
$$


In a similar manner, $p$ may be eliminated, giving

$\frac{\partial^{2} u}{\partial t^{2}}-a^{2} \frac{\partial^{2} u}{\partial x^{2}}=0$

The general solutions of Eqs.(9)- (12) are expressed as

$\left\{\begin{array}{l}p=R(t-x / a)+L(t+x / a) \\ \rho a u=R(t-x / a)-L(t+x / a)\end{array}\right.$

The function $R(t-x / a)$ expresses a wave propagating towards positive $x$ and $L(t+x / a)$ is another wave towards negative $x$. For the stability analysis, each quantity is separated into steady and unsteady components: $Q=\bar{Q}+\tilde{Q}(t), p=\bar{p}+\tilde{p}(t)$ and $u$ $=\bar{u}+\tilde{u}(t)$. The absolute value of steady part is assumed to be much larger than unsteady part. Only taking the unsteady part into account, the general solutions are assumed as:

$$
\begin{aligned}
& \tilde{p}=p_{R} \cdot e^{j \omega(t-x / a)}+p_{L} \cdot e^{j \omega(t+x / a)} \\
& \rho a \tilde{u}=p_{R} \cdot e^{j \omega(t-x / a)}-p_{L} \cdot e^{j \omega(t+x / a)}
\end{aligned}
$$

Solutions (13) and (14) satisfy Eqs.(11) and (12) generally even with a complex value of $\omega$. Assuming no pressure fluctuation ( $\tilde{p}=0)$ at the entrance of the inlet pipe $(x=0), p_{R}+p_{L}=0$ is obtained from Eq.(13). By putting this result back into Eqs.(13) and

(14), the pressure and velocity fluctuations along the inlet pipe are

$$
\begin{aligned}
& \tilde{p}=p_{R} e^{j \omega t}\left(e^{-j \omega x / a}-e^{j \omega x / a}\right)=-2 j \cdot p_{R} e^{j \omega t} \sin (\omega x / a) \\
& \rho a \tilde{u}=p_{R} e^{j \omega t}\left(e^{-j \omega x / a}+e^{j \omega x / a}\right)=2 \cdot p_{R} e^{j \omega t} \cos (\omega x / a)
\end{aligned}
$$

Combining Eqs.(15) and (16), the pressure fluctuation can be correlated with the velocity fluctuation. The pressure fluctuation $\tilde{p}_{L i}$ at the inlet of the runner $x=L_{i}$ can be correlated with the velocity fluctuation $\tilde{u}_{L i}$ :

$\tilde{p}_{L i}=-j \cdot \rho a \tan \left(\omega \frac{L_{i}}{a}\right) \cdot \tilde{u}_{L i}$

The pressure difference between the inlet and exit of runner can be presented by

$$
p_{L i}-p_{a}=\frac{\rho}{2} \zeta_{T} u_{L i}^{2}=\frac{\rho}{2} \zeta_{T}\left(\bar{u}_{L i}^{2}+2 \bar{u}_{L i} \tilde{u}_{L i}+\tilde{u}_{L i}^{2}\right)
$$

where $p_{a}$ is the pressure at the turbine discharge and $\zeta_{\mathrm{T}}$ is a coefficient which represents the effect of runner. The unsteady part is

$$
\tilde{p}_{L i}-\tilde{p}_{a}=\rho \zeta_{T} \bar{u}_{L i} \tilde{u}_{L i}=\frac{\rho \zeta_{T}}{A_{i}^{2}} \bar{Q}_{1} \tilde{Q}_{1}
$$

The unsteady part of Bernoulli's equation (2) applied to the draft tube is

$$
\tilde{p}_{a}=\tilde{p}_{\text {exit }}+\frac{\rho L_{e}}{A_{e}} j \omega \tilde{Q}_{2}+\frac{\rho\left(\zeta_{2}-D\right)}{A_{e}^{2}} \bar{Q}_{2} \tilde{Q}_{2}
$$

By applying Eqs.(18) and (19) into (17), it is obtained:

$-j \cdot \rho a \tan \left(\omega \frac{L_{i}}{a}\right) \cdot \frac{\tilde{Q}_{1}}{A_{i}}-\left(\frac{\rho L_{e}}{A_{e}} j \omega \tilde{Q}_{2}+\frac{\rho\left(\zeta_{2}-D\right)}{A_{e}^{2}} \bar{Q}_{2} \tilde{Q}_{2}\right)=\frac{\rho \zeta_{T}}{A_{i}^{2}} \bar{Q}_{1} \tilde{Q}_{1}$

which can be reduced to

$$
\tilde{Q}_{1}=-\frac{\frac{\rho L_{e}}{A_{e}} j \omega+\frac{\rho\left(\zeta_{2}-D\right)}{A_{e}^{2}} \bar{Q}_{2}}{\frac{\rho \zeta_{T}}{A_{i}^{2}} \bar{Q}_{1}+j \cdot \frac{\rho a}{A_{i}} \tan \left(\omega \frac{L_{i}}{a}\right)} \tilde{Q}_{2}
$$

The unsteady part of continuity equation (8) between upstream and downstream flow rates $Q_{1}$ and $Q_{2}$ is

$$
\tilde{Q}_{2}-\tilde{Q}_{1}=-\frac{\rho L_{e} C}{A_{e}} \frac{d^{2} \tilde{Q}_{2}}{d t^{2}}+\frac{\rho C}{A_{e}^{2}}\left(D-\zeta_{2}\right) \bar{Q}_{2} \frac{d \tilde{Q}_{2}}{d t}+\frac{2 \rho C \alpha \cot \beta_{2}}{S}\left(\frac{\cot \beta_{2}}{S} \bar{Q}_{1}-u_{2}\right) \cdot \frac{d \tilde{Q}_{1}}{d t}
$$

By applying Eq. (20) into (21) and using $\tilde{Q}_{1}=A_{i} \tilde{u}_{l}$, the system characteristic equation assuming the finiteness of sound velocity in the penstock is obtained:

$$
\begin{aligned}
& \left(\frac{\rho a}{A_{i}} \frac{\rho L_{e}}{A_{e}} j C\right) \omega^{2} \tan \left(\omega \frac{L_{i}}{a}\right) \\
& +\left[\frac{\rho \zeta_{T}}{A_{i}^{2}} \bar{Q}_{1} \cdot \frac{\rho L_{e} C}{A_{e}}+\frac{2 \rho C \alpha \cot \beta_{2}}{S}\left(\frac{\cot \beta_{2}}{S} \bar{Q}_{1}-u_{2}\right) \cdot \frac{\rho L_{e}}{A_{e}}\right] \omega^{2}-\left[\frac{\rho a}{A_{i}} \frac{\rho C}{A_{e}^{2}}\left(D-\zeta_{2}\right) \bar{Q}_{2}\right] \omega \tan \left(\omega \frac{L_{i}}{a}\right) \\
& +\left[-\frac{\rho L_{e}}{A_{e}} j+\frac{\rho \zeta_{T}}{A_{i}^{2}} \bar{Q}_{1} \cdot \frac{\rho C}{A_{e}^{2}}\left(D-\zeta_{2}\right) \bar{Q}_{2} j-\frac{2 \rho C \alpha \cot \beta_{2}}{S}\left(\frac{\cot \beta_{2}}{S} \bar{Q}_{1}-u_{2}\right) \cdot \frac{\rho\left(\zeta_{2}-D\right)}{A_{e}^{2}} \bar{Q}_{2} j\right] \omega
\end{aligned}
$$


$-j \cdot \frac{\rho a}{A_{i}} \tan \left(\omega \frac{L_{i}}{a}\right)+\left[-\frac{\rho\left(\zeta_{2}-D\right)}{A_{e}^{2}} \bar{Q}_{2}-\frac{\rho \zeta_{T}}{A_{i}^{2}} \bar{Q}_{1}\right]=0$

Equation (22) is a transcendential equation in terms of $\omega$. From the characteristic equation (22), the complex frequency $\omega$ $=\omega_{R}+j \omega_{I}$ can be determined. The expression $e^{j \omega t}=e^{j \omega_{R} t} \cdot e^{-\omega_{I} t}$ shows that the real part $\omega_{R}$ gives the frequency and the imaginary part $\omega_{I}$ the damping rate.

When $\left|\omega \frac{L_{i}}{a}\right| \square 1, \tan \left(\omega \frac{L_{i}}{a}\right) \approx \omega \frac{L_{i}}{a}$ and $j \cdot \frac{\rho a}{A_{i}} \tan \left(\omega \frac{L_{i}}{a}\right) \approx \frac{\rho L_{i}}{A_{i}} j \omega$. For this case, the characteristic equation is reduced to a third order equation, which has been obtained for incompressible flow in the penstock ${ }^{[1]}$.

Equation (21) can be written as

$\rho C \frac{L_{e}}{A_{e}} \frac{d^{2} \widetilde{Q}_{2}}{d t^{2}}+\rho C \frac{\zeta_{2}-D}{A_{e}^{2}} \bar{Q} \frac{d \widetilde{Q}_{2}}{d t}+\widetilde{Q}_{2}=\widetilde{Q}_{1}+2 \rho C \alpha \frac{\cot \beta_{2}}{S}\left(\frac{\cot \beta_{2}}{S} \bar{Q}-U_{2}\right) \frac{d \widetilde{Q}_{1}}{d t}$

If considering the case of $\widetilde{Q}_{1}=0$, negative damping occurs when $D>\zeta_{2}$. This is caused by the diffuser effect of the draft tube. The draft tube resonance frequency is given by

$$
\omega_{e}=\sqrt{\frac{A_{e}}{\rho L_{e} C}}
$$

\section{Results and discussion}

Due to the term of $j \cdot \frac{\rho a}{A_{i}} \tan \left(\omega \frac{L_{i}}{a}\right)$, the characteristic equation (22) is a transcendental equation, having higher order solutions. An iterative method is used to solve Eq.(22). The values of the parameters used for sample calculations are given in the nomenclature. These values are determined by considering a test facility at EPFL and used for sample calculation as standard values except for the parameters specified for each case. The value of the loss coefficient $\zeta_{T}$ representing the effect of the runner was determined by assuming that the applied head $H$ equals to $\left(\zeta_{T} / 2 g\right)\left(\bar{Q} / A_{i}\right)^{2}$. The value of the cavitation compliance $C$ was determined so that the frequency given by Eq.(24) becomes 0.16 times the rotational speed of the runner $f_{n}=12.5 \mathrm{~Hz}$. The swirl free flow rate $Q_{s f}$ which gives no swirl at the runner exit is calculated to be $Q_{s f}=S U_{2} \tan \beta_{2}=0.618 \mathrm{~m}^{3} / \mathrm{s}$.
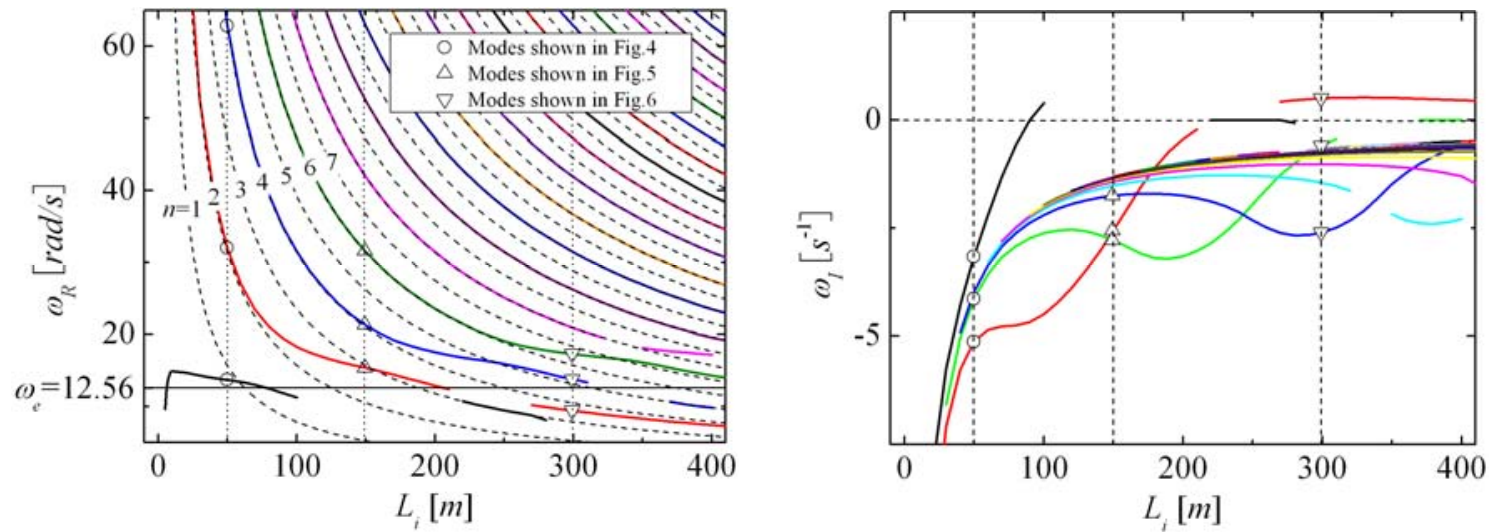

Fig. 3 Higher order frequencies under $a=500 \mathrm{~m} / \mathrm{s}$

\subsection{Effect of inlet pipe length $L_{i}$}

Figure 3 shows the solutions when the wave speed is $a=500 \mathrm{~m} / \mathrm{s}$ for the standard condition shown in the nomenclature. The mean flow rate $\bar{Q}=0.51 \mathrm{~m}^{3} / \mathrm{s}$ is smaller than the swirl free flow rate $Q_{s f}=0.618 \mathrm{~m}^{3} / \mathrm{s}$. The full line curves represent different order modes. The draft tube resonant frequency $\omega_{e}=\sqrt{A_{e} /\left(\rho L_{e} C\right)}=12.56 \mathrm{rad} / \mathrm{s}$ is also shown in Fig.3. The multiple quarter wavelength resonance frequencies $\omega_{n}=2 \pi n a / 4 L_{i}$ of the penstock are also plotted as dashed lines. The followings are observed in the figure:

1) At frequencies higher than about twice the draft tube frequency $2 \omega_{e}$, the frequency obtained agrees with $\omega_{n}$ with even number of $n$. It will be shown later that these correspond to open-to-open resonant frequencies of the penstock.

2) As the frequencies approach the draft tube resonant frequency $\omega_{e}$, the obtained frequencies deviates from $\omega_{n}$ in the direction away from $\omega_{e}$. This is opposite to the "lock in" phenomena observed for Karman vortex from flexibly supported cylinders.

3) When $\bar{Q}<Q_{s f}$, most of the modes have negative damping $\left(\omega_{I}<0\right)$.

At $L_{i}=50 \mathrm{~m}, 150 \mathrm{~m}$ and $300 \mathrm{~m}$, the lowest 3 mode frequencies and corresponding velocity and pressure fluctuation modes are shown in Fig. 4 to $6 . \mathrm{x}=0$ corresponds to the penstock inlet and $\mathrm{x}=\mathrm{Li}$ is the exit to runner. Instantaneous velocity and pressure fluctuations are plotted to show the mode of oscillations corresponding to each eigen frequency, At $L_{i}=50 \mathrm{~m}$, the fluctuations of $1^{\text {st }}$ mode has about $1 / 4$ wavelength, and $2^{\text {nd }}$ and $3^{\text {rd }}$ order are about $1 / 2$ and 1 wavelength respectively, as expected from the 
comparison with the multiple quarter wavelength frequencies shown in Fig. 3 . At $L_{i}=50 \mathrm{~m}$ and $150 \mathrm{~m}$, for the $1^{\text {st }}$ order mode with the frequency closer to $\omega_{e}$, the runner inlet is a node of velocity fluctuation. However, the runner inlet is a loop for higher order modes. At $L_{i}=300 \mathrm{~m}$, the runner inlet is a node of velocity fluctuation for the $2^{\text {nd }}$ order mode, whose frequency is closer to $\omega_{e}$ than other order frequencies. The $1^{\text {st }}$ order mode with the frequency less than $\omega_{e}$ is a damping mode as shown in Fig. 3.
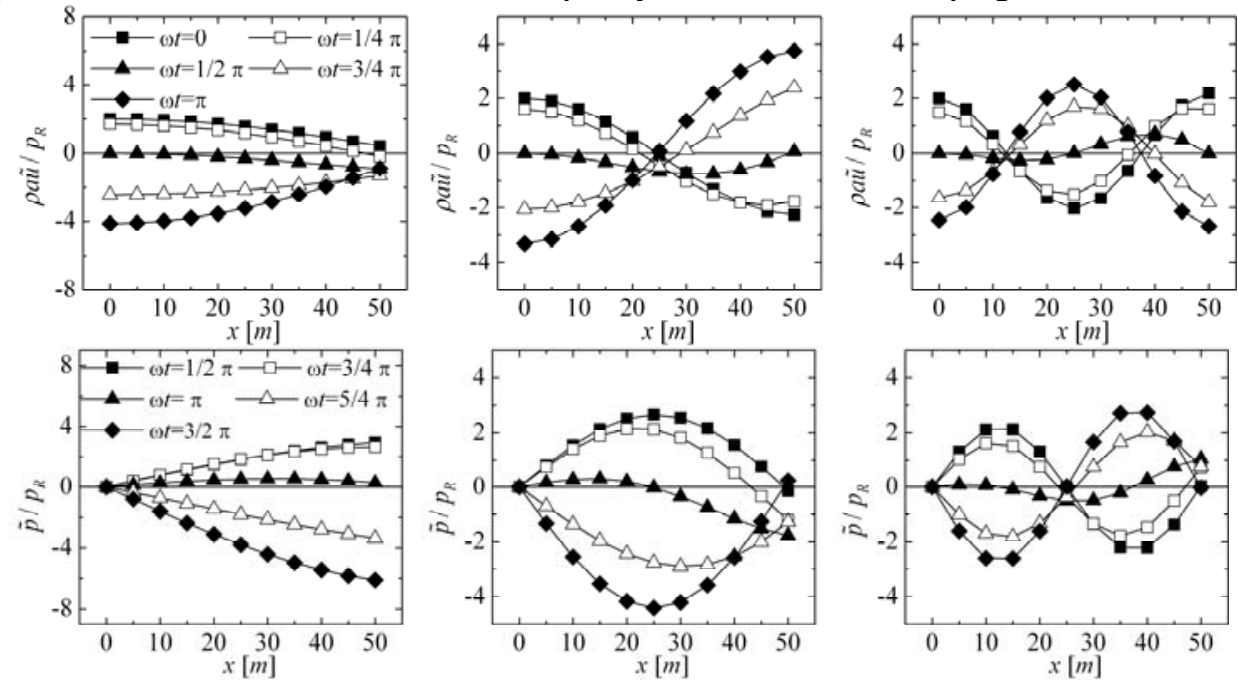

$\omega_{1}=13.683-3.158 \mathrm{j}$

$\omega_{2}=31.909-5.126 \mathrm{j}$

$\omega_{3}=62.937-4.140 \mathrm{j}$

Fig. 4 Velocity and pressure fluctuations along the inlet pipe at $L_{i}=50 \mathrm{~m}$
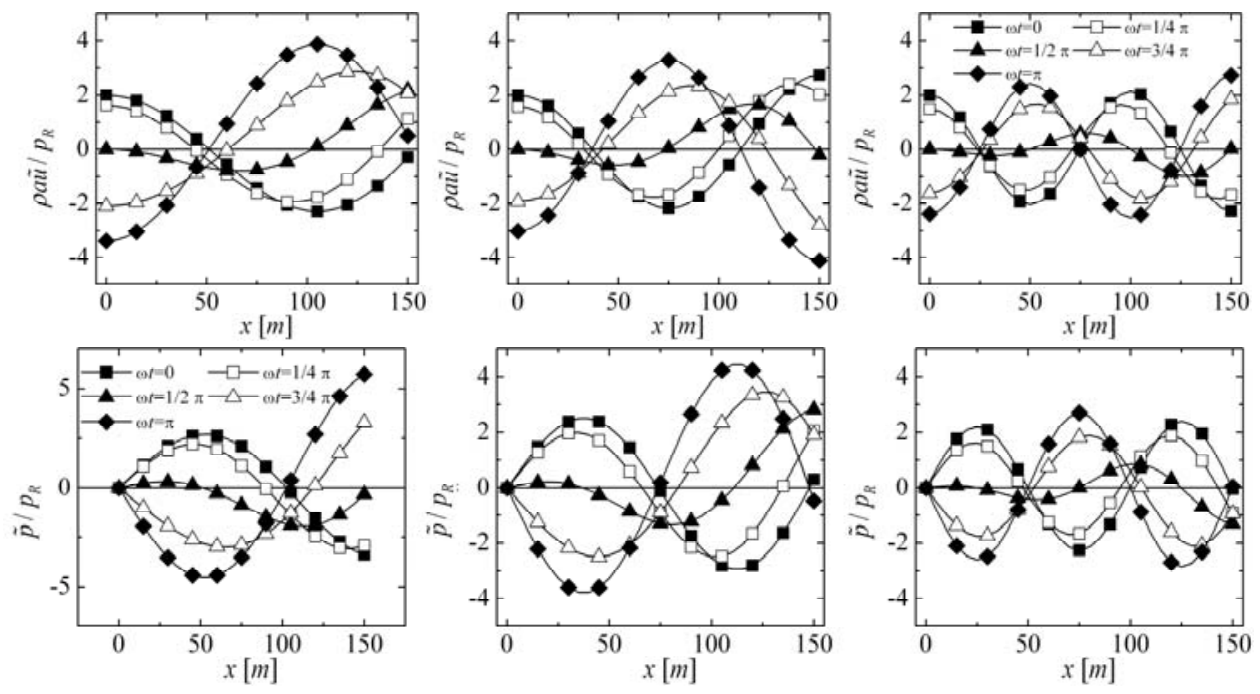

$\omega_{1}=15.335-2.561 \mathrm{j}$

$\omega_{2}=21.256-2.787 \mathrm{j}$

$\omega_{3}=31.465-1.753 \mathrm{j}$

Fig. 5 Velocity and pressure fluctuations along the inlet pipe at $L_{i}=150 \mathrm{~m}$
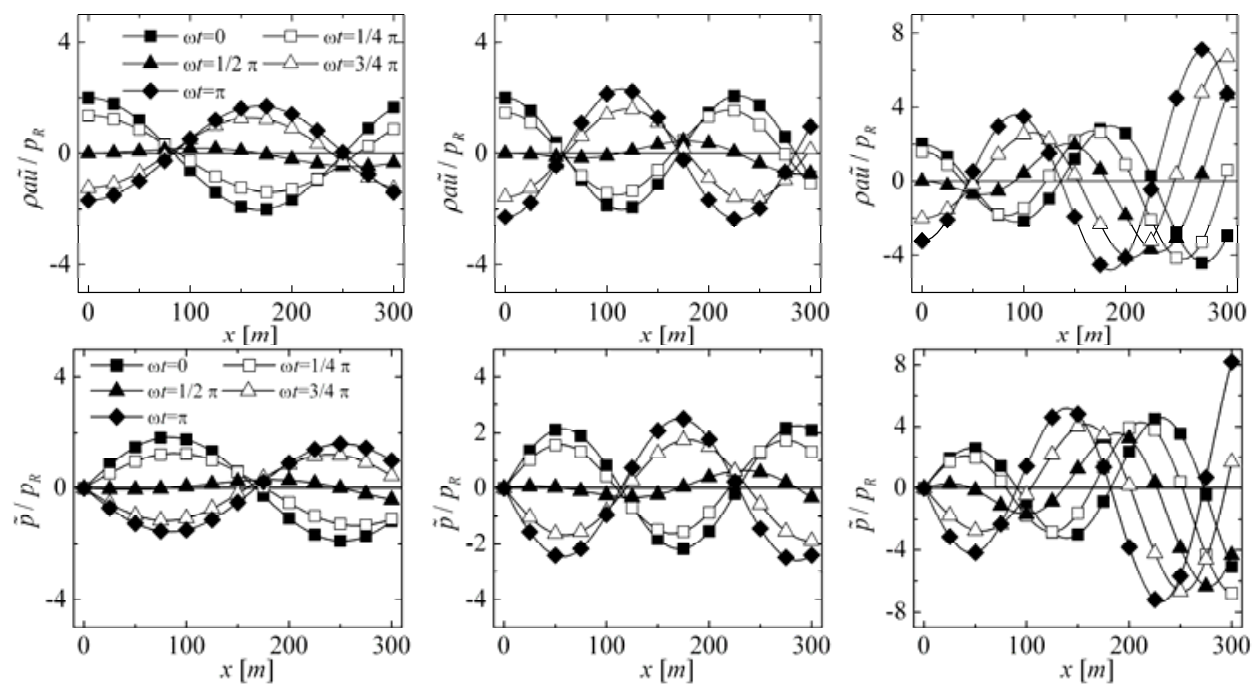

$\omega_{1}=9.381+0.504 \mathrm{j}$

$\omega_{2}=13.763-0.597 \mathrm{j}$

$\omega_{3}=17.274-2.606 \mathrm{j}$

Fig. 6 Velocity and pressure fluctuations along the inlet pipe at $L_{i}=300 \mathrm{~m}$ 


\subsection{Effects of mean flow rate and contributions of diffuser and swirl effects}

The lowest order mode with the frequency close to $\omega_{e}$ for $L_{i}=50 \mathrm{~m}$ is considered firstly. Figure 7 shows the results under standard condition with three different values of wave speed $a$. Although some effects of the wave speed are observed, general characteristics are not affected by the wave speed.

Figure 8 shows the effects of mean flow rate on the lowest 3 frequencies under $a=500 \mathrm{~m} / \mathrm{s}$. The real parts of $2^{\text {nd }}$ and $3^{\text {rd }}$ order frequencies $\omega_{2}$ and $\omega_{3}$ increase as the flow rate departs from the swirl free flow rate $Q_{s f}$. While $\omega_{1}$ shows negative damping in a wider region of mean flow rate, $\omega_{2}$ and $\omega_{3}$ have negative damping in the region of $\bar{Q}<Q_{s f}$.

The diffuser and swirl effects are examined separately by putting $\alpha=0$ or $D-\zeta_{2}=0$. Figure 9 shows the results for $L i=50 \mathrm{~m}$ and $a=500 \mathrm{~m} / \mathrm{s}$. With $\alpha=0, \omega_{2}$ and $\omega_{3}$ are always damping $\left(\omega_{2}, \omega_{3}>0\right)$ without the effect of $D$ value. This shows that the diffuser effect does not cause higher order modes (with $\omega_{1}, \mathrm{i} \geq 2$ ) in the penstock. With $D-\zeta_{2}=0$ and larger values of $\alpha$, all modes have negative / positive damping when $\bar{Q}</>Q_{s f}$. This shows that the swirl effect affects all modes and causes instabilities when $\bar{Q}<Q_{s}$.
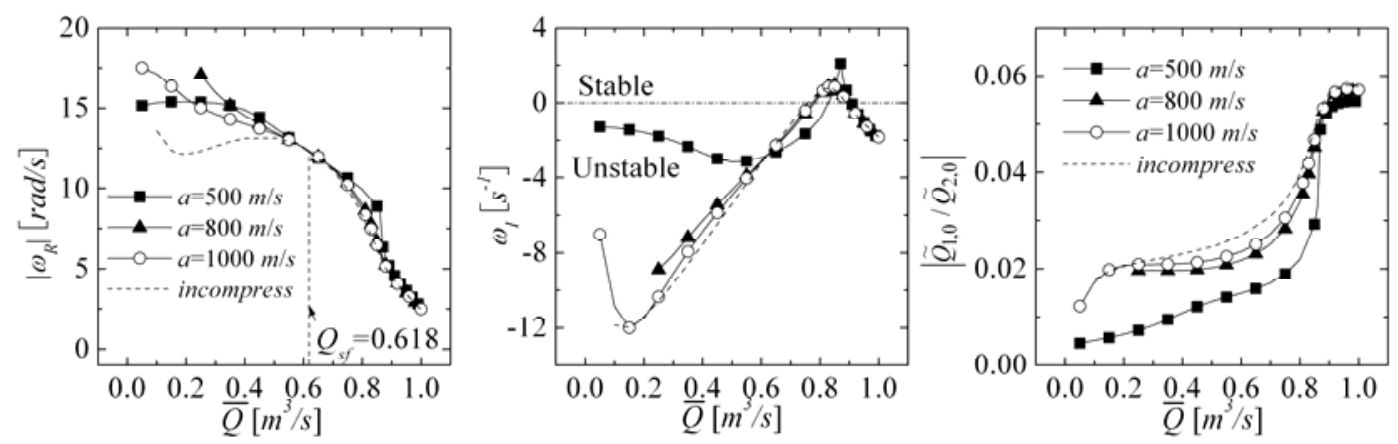

Fig. 7 Effect of mean flow rate $\bar{Q}$ under standard condition $\left(L_{i}=50 \mathrm{~m}\right)$
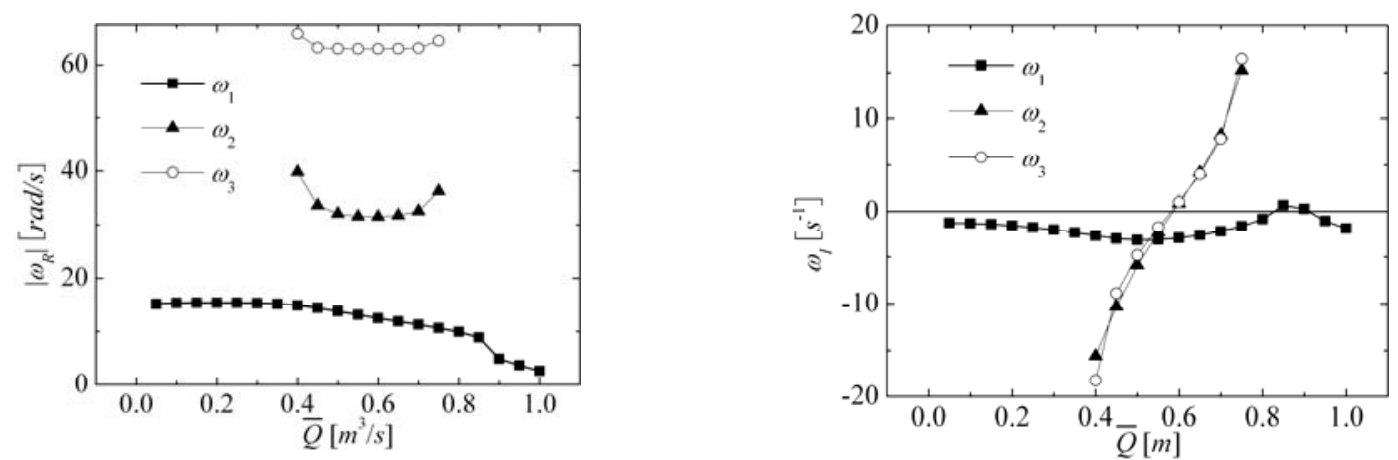

Fig. 8 Effect of $\bar{Q}$ to first 3 order frequencies at $L i=50 \mathrm{~m}$, under $a=500 \mathrm{~m} / \mathrm{s}$

Table 1 First 3 order frequencies and mode under $\alpha=0, D=0.5 D_{\text {stand, }}$,

\begin{tabular}{c|c|c|c|c|c|c}
\hline \multirow{2}{*}{ order } & \multicolumn{2}{|c|}{$L_{i}=50 \mathrm{~m}$} & \multicolumn{2}{c|}{$L_{i}=150 \mathrm{~m}$} & \multicolumn{2}{c}{$L_{i}=300 \mathrm{~m}$} \\
\cline { 2 - 7 } & $\omega$ & $\tilde{Q}_{1,0} / \tilde{Q}_{2,0}$ & $\omega$ & $\tilde{Q}_{1,0} / \tilde{Q}_{2,0}$ & $\omega$ & $\tilde{Q}_{1,0} / \tilde{Q}_{2,0}$ \\
\hline 1 & $12.574-1.151 \mathrm{j}$ & $-0.010-0.006 \mathrm{j}$ & $12.657-1.004 \mathrm{j}$ & $-0.0232-0.0299 \mathrm{j}$ & $10.375+0.324 \mathrm{j}$ & $0.3239-0.1991 \mathrm{j}$ \\
\hline 2 & $31.594+2.544 \mathrm{j}$ & $-5.242-1.495 \mathrm{j}$ & $21.059+0.838 \mathrm{j}$ & $-1.7912-0.5412 \mathrm{j}$ & $12.536-1.065 \mathrm{j}$ & $-0.0041-0.0199 \mathrm{j}$ \\
\hline 3 & $62.912+2.563 \mathrm{j}$ & $-23.983-2.991 \mathrm{j}$ & $31.476+0.852 \mathrm{j}$ & $-5.2567-0.8144 \mathrm{j}$ & $15.824+0.386 \mathrm{j}$ & $-0.5789-0.3161 \mathrm{j}$ \\
\hline
\end{tabular}

The effects of swirl and diffuser at $L i=150 \mathrm{~m}$ are shown in Fig.10. Figure 3 shows that the lowest order frequency $\omega_{1}$ at $L i$ $=150 \mathrm{~m}$ corresponds to the second order frequency $\omega_{2}$ at $L i=50 \mathrm{~m}$. This is also shown in Figs. 4 and 5 for the $1 \mathrm{st}$ order mode. However, comparing Figs. 9 and 10, the results of $L i=150 \mathrm{~m}$ for $\omega_{1}$ are similar to the results for $\omega_{1}$ of $L i=50 \mathrm{~m}$, with the values of $\omega_{1}$ close to $\omega_{e}$ for both cases. The diffuser still has a significant effect on $\omega_{1}$ but little on $\omega_{2}$ and $\omega_{3}$. This shows that the diffuser effect affects the modes with the resonant frequency close to $\omega_{e}$, independently on the mode shape in the penstock. On the other hand, swirl has larger effect on all of the lowest 3 frequency modes.

The smaller diffuser effect on higher order frequencies may be explained by the draft tube resonance frequency $\omega_{\mathrm{e}}=12.56 \mathrm{~Hz}$. Table 1 shows $\tilde{Q}_{1,0} / \tilde{Q}_{2,0}$ for each case with the values of $\omega$. $\tilde{Q}_{1,0}$ and $\tilde{Q}_{2,0}$ are flow rate fluctuations at the runner inlet and outlet. For the cases with $\omega_{1}$ for $L_{i}=50 \mathrm{~m}$ and $150 \mathrm{~m}$ and $\omega_{2}$ for $L_{i}=300 \mathrm{~m}$, the frequencies are closer to $\omega_{e}$. For these cases $\left|\tilde{Q}_{1,0} / \tilde{Q}_{2,0}\right|$ has smaller values suggesting that oscillations in the draft tube is much larger. For these cases the effects of $D$ is significant. For other cases, $\tilde{Q}_{1,0} / \tilde{Q}_{2,0}$ has larger values suggesting larger amplitude of oscillations in the penstock. 


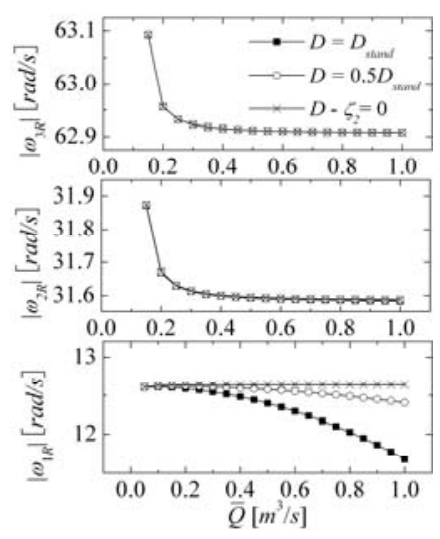

a) $\alpha=0$

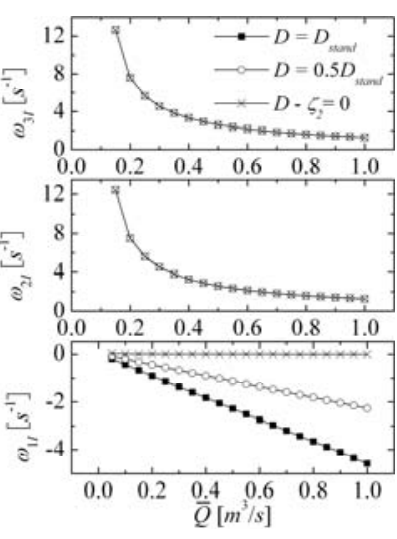

Fig. 9 Diffuser and swirl effects to lowest 3 frequencies at $L i=50 \mathrm{~m}, a=500 \mathrm{~m} / \mathrm{s}$

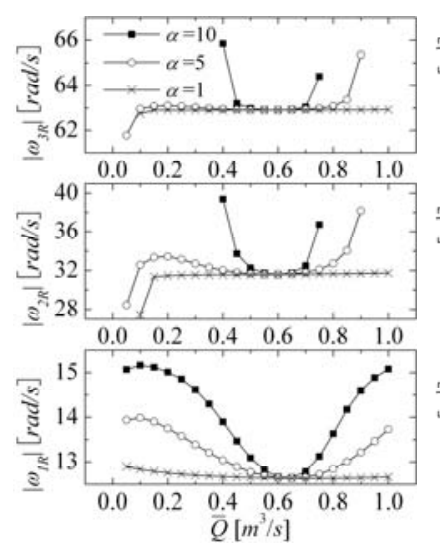

b) $D-\zeta_{2}=0$

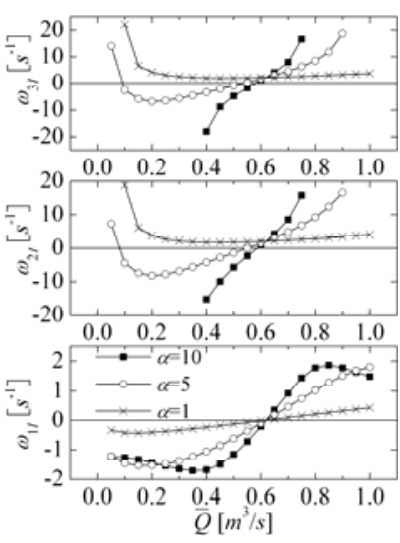

$Q[\mathrm{~m} / \mathrm{s}]$

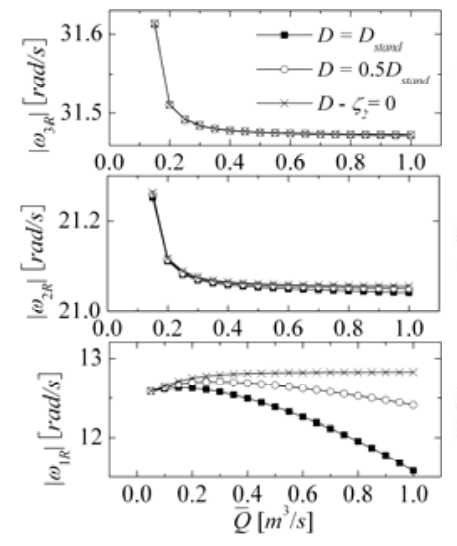

a) $\alpha=0$
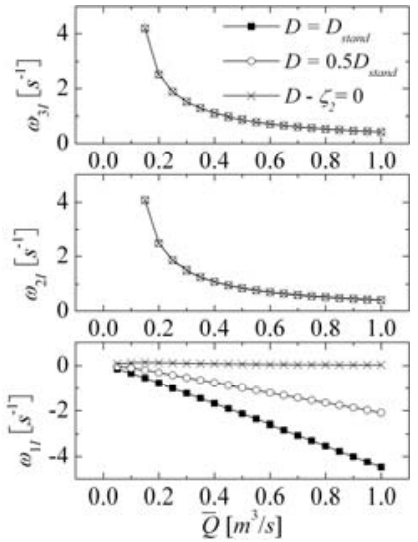

$\bar{Q}\left[m^{3} / s\right]$
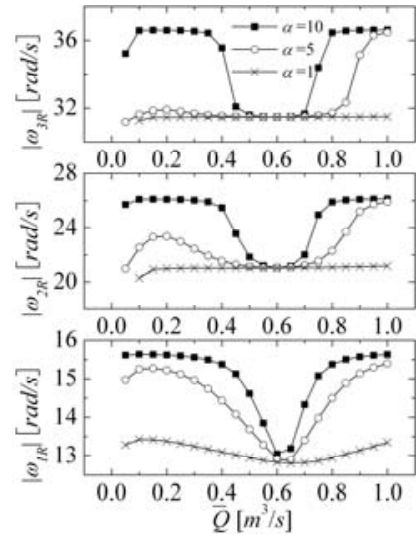

b) $D-\zeta_{2}=0$
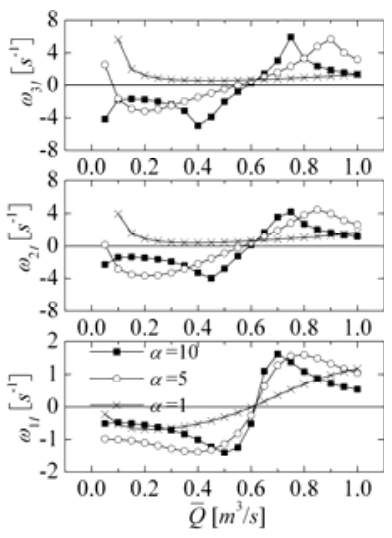

$\bar{Q}\left[\mathrm{~m}^{3} / \mathrm{s}\right]$

Fig. 10 Diffuser and swirl effects to lowest 3 order frequencies at $L i=150 \mathrm{~m}, a=500 \mathrm{~m} / \mathrm{s}$

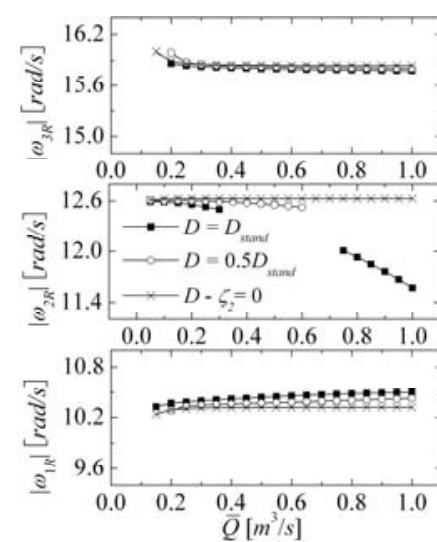

a) $\alpha=0$
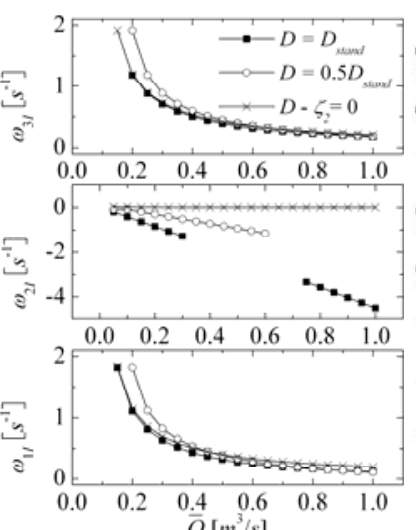

$Q[\mathrm{~m} / \mathrm{s}]$
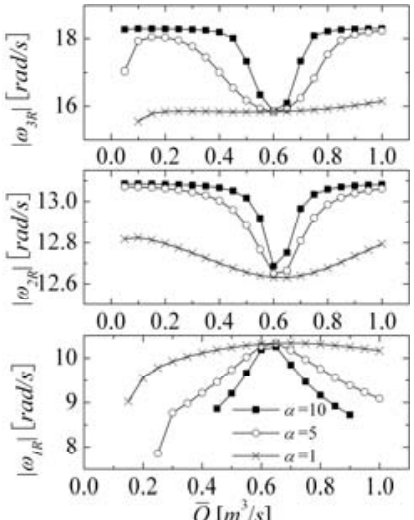

b) $D-\zeta_{2}=0$

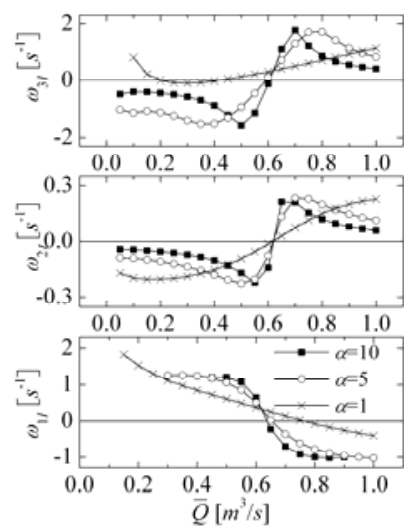

Fig. 11 Diffuser and swirl effects to lowest 3 order frequencies at $\mathrm{Li}=300 \mathrm{~m}, a=500 \mathrm{~m} / \mathrm{s}$

The diffuser and swirl effects are also checked at $L_{i}=300 \mathrm{~m}$ in Fig. 11 . The diffuser has larger effect on $\omega_{2}$ but little on $\omega_{1}$ and $\omega_{3}$. This is because $\omega_{2}$ has the nearest value to the draft tube resonance frequency $\omega_{e}$ at $L_{i}=300 \mathrm{~m}$. The swirl effect on the $1^{\text {st }}$ order mode with the frequency less than $\omega_{e}$ is totally different from other cases. This will be discussed later.

Comparing Figs.9 to 11, for the diffuser effects $(\alpha=0)$, only the modes with the frequency closest to the draft tube resonant frequency $\omega_{e}$ are affected and the instability is caused. Other modes with the frequencies far from $\omega_{e}$ are not affected nor destabilized by the diffuser effect.

On the other hand, the swirl effects $\left(D-\zeta_{2}=0\right)$ affects all modes except for the first order mode of $L_{i}=300 \mathrm{~m}$, which has a resonant frequency lower than $\omega_{e}$, and destabilizes them at $\bar{Q}<Q_{s f}$. The destabilizing swirl effect for the general case is explained as follows. Consider the case when the upstream flow rate $Q_{1}$ increases under the mean flow rate $\bar{Q}</>Q_{s f}$. Then the swirl velocity decreases / increases and the pressure in the vortex core is increased / decreased. This results in decreased / increased cavity volume $V_{C}$. From the continuity equation $d V_{C} / d t=Q_{2}-Q_{1}, Q_{2}$ will be decreased / increased and $Q_{1}$ will be increased / decreased for the general cases. This means that the cavity volume fluctuation provides positive / negative feed back at 
the mean flow rate $\bar{Q}$ smaller / larger than the swirl free flow rate $Q_{s f}$.

Figure 12 shows the plot of $\tilde{Q}_{1,0} / \tilde{Q}_{2,0}$ for the cases of $D-\zeta_{2}=0, \alpha=5, L_{i}=150 \mathrm{~m}$ and $300 \mathrm{~m}$. Almost for all cases, $\left|\tilde{Q}_{1,0} / \tilde{Q}_{2,0}\right| \square 1$. For these cases, the cavity volume fluctuation $V_{C}$ is delayed behind the $\tilde{Q}_{2}$ fluctuation by about $90^{\circ}$, from the continuity relation $\tilde{Q}_{2}=d V_{c} / d t$. Except for the case of $\omega_{1}<\omega_{e}$ with $L_{i}=300 \mathrm{~m}, \operatorname{Real}\left(\tilde{Q}_{1,0} / \tilde{Q}_{2,0}\right)<0$ and the phase difference between $\tilde{Q}_{1}$ and $\tilde{Q}_{2}$ is larger than $\pm 90^{\circ}$. For this case upstream flow rate is decreased $\left(\tilde{Q}_{1}<0\right)$ when the cavity volume is increasing $\left(d V_{c} / d t>0\right)$, as assumed in the discussion above. For the case of $\omega_{1}<\omega_{e}$ with $L_{i}=300 \mathrm{~m}, \operatorname{Real}\left(\tilde{Q}_{1,0} / \tilde{Q}_{2,0}\right)>0$ and the phase difference between $\tilde{Q}_{1}$ and $\tilde{Q}_{2}$ is smaller than $\pm 90^{\circ}$. In this case, both upstream and downstream flow rates becomes positive when $d V_{c} / d t>0$. This is opposite to the assumption in the above discussion. The positive feedback and the instability occur for the flow rates larger than the swirl free flow rate. The relationship between the cavity volume and flow rate fluctuation is shown in Fig. 13 for the typical cases with phase difference equals to $180^{\circ}$ (general cases) or $0^{\circ}$ (for $\omega_{1}$ with $L_{i}=300 \mathrm{~m}$ ).

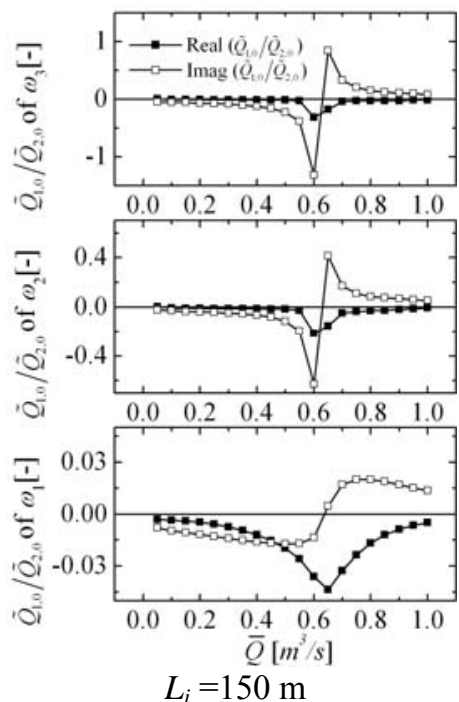

Fig. 12 Modes of first 3 order frequencies at $D-\zeta_{2}=0$ and $\alpha=5$

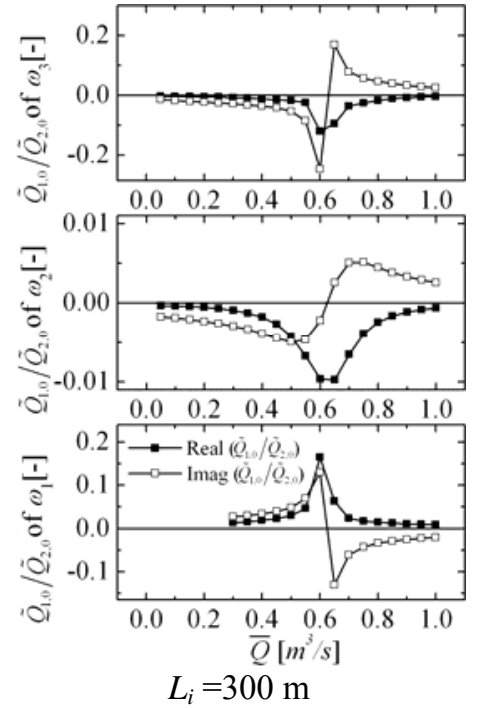

Fig. 13

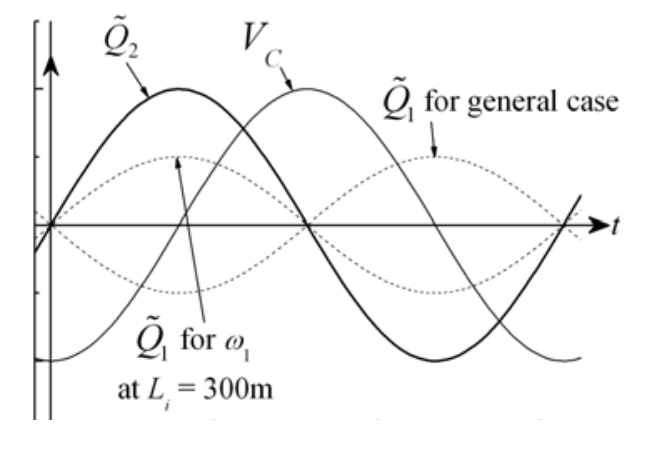

Fig. 13 Relationship of flow rate fluctuations and cavity volume

\section{Conclusion}

It was shown that various acoustic modes in the penstock can be destabilized by diffuser and swirl effects. At higher frequency than the draft tube resonant frequency $\omega_{e}$, open-to-open acoustic modes occur in the penstock, when the flow rate is smaller than swirl free flow rate $Q_{s f}$. When the frequency of acoustic modes gets closer to $\omega_{e}$, the oscillation frequency has a tendency to depart from $\omega_{e}$. The diffuser effect of draft tube affects only the modes with the frequency closest to $\omega_{e}$ and causes the instability at all flow rates. For these modes, the flow rate fluctuation in the upstream of the runner is much smaller than the downstream and the velocity node occurs at the runner inlet. The swirl effect affects all modes and cause instability at smaller flow rates than $Q_{s f}$, except for the mode with the frequency less than $\omega_{e}$. Thus, the higher order modes are caused by swirl effect. For the modes with the frequency less than $\omega_{e}$, the phase difference between upstream and downstream flow rate fluctuation becomes less than $90^{\circ}$ and the instability occurs at higher flow rate than $Q_{s f}$.

\section{Nomenclature}

$a=500 \mathrm{~m} / \mathrm{s}$
$A_{c}=0.125 \mathrm{~m}^{2}$
$A_{e}=0.125 \mathrm{~m}^{2}$
$A_{i}=0.22 \mathrm{~m}^{2}$
$C=97.2 \times 10^{-7} \mathrm{~m}^{4} \mathrm{~s}^{2} / \mathrm{kg}$
$D=\left(A_{e} / A_{c}\right)^{2}-1=27.7$
$H=14.8 \mathrm{~m}$
$L_{e}=4.36 \mathrm{~m}$
$L_{i}=50 \mathrm{~m}$
$p$
$Q=0.51 \mathrm{~m}^{3} / \mathrm{s}$
$Q_{s f}=0.618 \mathrm{~m}^{3} / \mathrm{s}$

$a=500 \mathrm{~m} / \mathrm{s}$

$A_{c}=0.125 \mathrm{~m}^{2}$

$A_{e}=0.125 \mathrm{~m}^{2}$

$A_{i}=0.22 \mathrm{~m}^{2}$

$C=97.2 \times 10^{-7} \mathrm{~m}^{4} \mathrm{~s}^{2} / \mathrm{kg}$

$H=14.8 \mathrm{~m}$

$L_{e}=4.36 \mathrm{~m}$

$L_{i}=50 \mathrm{~m}$

$Q=0.51 \mathrm{~m}^{3} / \mathrm{s}$

$Q_{s f}=0.618 \mathrm{~m}^{3} / \mathrm{s}$
Wave speed in the penstock

Draft tube inlet area

Draft tube exit area

Inlet pipe sectional area

Cavitation compliance

Diffusion factor of draft tube

Head

Effective length of draft tube

Inlet pipe length

Pressure

Flow rate

Swirl free flow rate

$$
\begin{aligned}
& S=0.125 \mathrm{~m}^{2} \\
& u \\
& U_{2}=15.7 \mathrm{~m} / \mathrm{s} \\
& V_{c} \\
& \alpha=10 \\
& \beta_{2}=17.5^{\circ} \\
& \rho=1000 \mathrm{~kg} / \mathrm{m} 3 \\
& \omega=\omega_{R}+j \omega_{I} \\
& \omega_{e}=12.56 \mathrm{rad} / \mathrm{s} \\
& \zeta_{2}=0.207 \\
& \zeta_{T}=54.2
\end{aligned}
$$

Runner exit area Flow velocity

Runner exit circumferential velocity Volume of cavity

Pressure coefficient of swirl Runner exit blade angle

Fluid density

Draft tube resonance frequency

Complex frequency

Loss coefficient of draft tube

Runner resistance 


\section{References}

[1] Chen, C., Nicolet, C., Yonezawa, K., Farhat, M., Avellan, F., Tsujimoto, Y., 2008, “One-Dimensional Analysis of Full Load Draft Tube Surge", ASME Trans. J. Fluids Eng., 130, 041106(2008).

[2] Jacob, T., Prenat, J-E., 1996, "Francis Turbine Surge: Discussion and Data Base", Proc. 18th IAHR Symposium, Valencia, Spain.

[3] Nishi, M., 1984, "Surging Characteristics of Conical and Elbow Type Draft Tubes", Pro. 12th IAHR Symposium on Hydraulic Machinery and System, Stirling, pp. 272-283.

[4] Nishi, M., Matsunaga, S., Kubota, T., Senoo, Y., 1982, "Flow Regimes in an Elbow-Type Draft Tube", Proc. 11th IAHR Symposium on Hydraulic Machinery and System, Amsterdam, pp. 1-13, paper 38.

[5] Nishi, M., Wang, X., Okamoto, M., Matsunaga, S., 1994, "Further Investigation on the Pressure Fluctuations Caused by Cavitated Vortex Rope in an Elbow Draft Tube", Cavitation and Gas Fluid Flow Machinery and Devices, ASME, pp. 63-70.

[6] Koutnik, J., Pulpitel, L., "Modeling of the Francis Turbine Full-Load Surge”, Modeling, Testing and Monitoring for Hydro Power Plants, Lausanne, 1996.

[7] Koutnik, J., Nicolet, C., A.Schoul, G., Avellen, F., “Overload Surge Event in a Pumped- Storage Power Plant”. In Proceeding of the 23rd IAHR Symposium, Yokohama, 2006, paper 135.

[8] Brennen, C.E., 1994, Hydrodynamics of Pumps, Concepts ETI, Inc \& Oxford Univ. Press, pp. 198-199.

[9] Susan-Resiga, R., Ciocan, G.D., Anton, I., Avellan, F., 2006, "Analysis of the Swirling Flow Downstream a Francis Turbine Runner", J. Fluid Eng., 128, pp. 177-189. 